\title{
Conservation of a Threatened Indigenous Fowl (Kureko Dori) Using the Germline Chimeras Transplanted from Primordial Germ Cells
}

\author{
Takashi Kuwana ${ }^{1}$, Takaharu Kawashima ${ }^{1}$, Mitsuru Naito², Hiroaki Yamashita ${ }^{3}$, \\ Masaharu Matsuzaki ${ }^{3}$ and Toshinori Takano ${ }^{3}$ \\ ${ }^{1}$ Laboratory of Intellectual Fundamentals for Environmental Studies, National Institute \\ for Environmental Studies, Tsukuba, Ibaraki 305-8506, Japan \\ ${ }^{2}$ Animal Genetic Engineering Laboratory, National Institute of Agrobiological Sciences, \\ Tsukuba, Ibaraki 305-8602, Japan \\ ${ }^{3}$ Kumamoto Prefectural Agricultural Research Center, Animal Husbandry Research Institute, \\ Kumamoto 861-1113, Japan
}

This is the first successful report on producing the endangered domestic fowl, Kureko Dori (KD), which is a Kumamoto prefectural natural treasure, using germline chimeras transplanted from primordial germ cells (PGCs) in practice. The attempt was made to produce germline chimeras between KD fowl, as a model of rare and/or endangered poultry, and common White Leghorn (WL) for the proliferation of KD individuals. The PGCs of KD were collected from embryonic blood at stages 13 to $16(\mathrm{H} \& \mathrm{H})$ and preserved in liquid nitrogen until injection. A certain number of frozen-thawed PGCs were picked up under a phase contrast microscope, and injected into the peripheral vein of WL embryos (common poultry) at stages 12 to 14 . We obtained one male and 6 females as the putative germline chimera, they were raised until sexual maturity, and the progeny test was performed by mating them with KD of the opposite sex to analyze the efficiency of germline chimera. The average rate of offspring originating from transplanted PGCs of KD was only $1.4 \%$, and the maximum was $5.3 \%$ from all 577. These KD offspring derived from the KD-WL germline chimeras also achieved fertility as normal. These results show that rare and/or endangered birds can proliferate through producing germline chimeras between the same species of bird.

Key words : endangered species, domestic fowl, germline chimera, primordial germ cells, transplantation

\section{Introduction}

Kureko Dori (KD) is an endangered domestic fowl breed in the Kureko region of Kumamoto prefecture in Japan. With strict screening criteria, KD was designated a prefectural natural treasure in 1965. So called "Nostril Processes" and a silver laced feather color are characteristic phenotypes of KD (Fig. 1). It might be considered that the processes are expressed by the breda comb gene (bd), which is very rare in the world (Matsuzaki, 2001). In order to rescue and maintain the KD breed, we considered that the procedures of experimental embryology with primordial germ cells (PGCs) might be useful to prevent extinction. This is the first successful report on the production of rare and/or endangered avian individuals using germline chimeras from transplanted PGCs in practice.

PGCs are the original cells of future functional

Received : August 17, 2005, Accepted : September 21, 2005

Address correspondence to: Takashi Kuwana, Ph. D., Laboratory of Intellectual Fundamentals for Environmental Studies, National Institute for Environmental Studies, 16-2 Onogawa Tsukuba, Ibaraki 305-8506, Japan

Tel : +81-29-850-2294 Fax : +81-29-850-2673 E-mail address : kuwana@nies.go.jp 


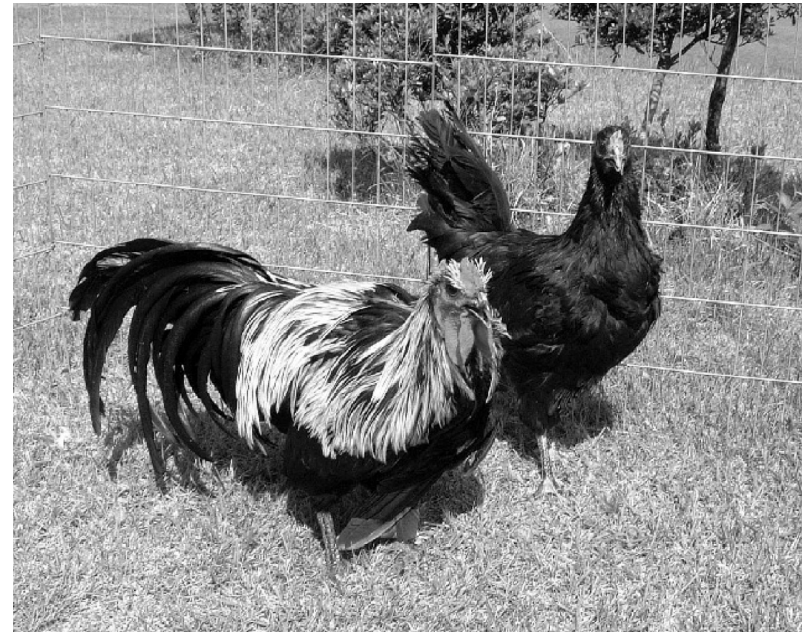

Fig. 1. Adult Kureko Dori (KD). The male KD (left) has the characteristic phenotype of a steady, silver laced feather color. The right is a female KD.

gametes, similar to oocytes or sperm in the early embryonic stages. They are usually initially identified at a region far from the future gonads, and migrate toward the gonadal anlage as their target organ to differentiate into functional gametes (Kuwana, 1993). The localization of avian embryo PGCs at the primitive streak stage was first described by Swift (1914). During this period, they are distributed on the extraembryonic endoblast in the form of a "germinal crescent" anterior and lateral to the embryo at the junction of the area pellucida and vitelline wall. They gradually circulate with embryonic blood circulation along with the establishing blood vascular system, just before the passive trapping of the capillary network at the vicinity of the presumptive gonads because of their larger diameter compared to blood cells. Then, they interstitially migrate into the germinal epithelium and differentiate into definitive gametes (Kuwana, 1993 ; Kuwana and Rogulska, 1999).

Avian PGCs undergo a period of migration using the blood vascular system, and this migration step is a suitable time for their isolation and manipulation. Chicken PGCs during their circulating phase were isolated from embryonic blood, and purified by Ficoll density gradient centrifugation (Yasuda et al., 1992). Tajima et al. (1993) produced germline chimeras by injecting such purified PGCs into the blood vessel of another chicken embryo. Hatched chickens were obtained; both males and females were raised until sexual maturity. Offspring apparently derived from the transferred PGCs, as judged by their feather color, were produced in the progeny test. These results support the idea that PGCs injected surgically into the vascular system of recipient embryos are capable of differentiating normally into either spermatogonia or oogonia in response to the gonadal environment of the recipient embryos.

Recently, Natio et al. (1994 a) reported the results of producing a highly efficient gonadal chimera using White Leghorn (WL) and Rhode Island Red breeds. Thus, these techniques appear to provide a promising means of conservation if successfully applied to rare and/or endangered poultry. However, in all these cases, popular and general fowl breeds were used as donors and recipients for the producing gonadal chimeras, i.e. WL, Rhode Island Red, Barred Plymouth Rock and so on. No attempt has been made to produce germline chimera injected with PGCs using other poultry including rare and/or endangered species. In the present study, we attempted to produce germline chimera between $\mathrm{KD}$, as a model of an endangered chicken breed, and WL, as a common poultry, to obtain offspring maintaining normal reproductive abilities.

\section{Materials and Methods}

\section{Breeds of Fowl}

Fertilized eggs of the KD breed were obtained by artificial insemination from genetic stocks maintained at the Kumamoto Prefectural Agricultural Research Center (Kikuchi-gun, Japan). Also, fertilized eggs of the WL breed were purchased from a commercial source (Aramaki Chicken Egg Farm, Ohita, Japan). All animals received humane care as outlined in the Guide for the Care and Use of Experimental Animals (Kumamoto Prefectural Agricultural Research Center Animal Care Committee).

\section{Preparation of PGCs}

Fertilized KD eggs were incubated at $38.5^{\circ} \mathrm{C}$ with approximately $60 \%$ relative humidity in a forced air incubator (Model P-008, Showa Incubator Laboratory, Saitama, Japan) for 52 to $58 \mathrm{hrs}$ to obtain embryos stages 13 to 16 (Hamburger and Hamilton, 1951). After cracking the eggshells, incubated KD eggs were transferred into petri dishes and embryonic blood was collected from the dorsal aorta and peripheral vein with a fine glass 
micropipette (a disposable $1.0 \mathrm{~mm}$-outside and 0.75 $\mathrm{mm}$-inside diameter pipette, Sutter Instrument Co., CA, USA, used after drawing out using a tiny flame ; the inner diameter of the drawn tip was about 50 $\mu \mathrm{m})$. In order to measure the volume of collected blood, a mark was drawn on the glass pipette up to the same level where the blood was sucked into the pipette. The volume of collected blood was estimated by weighing the same amount of distilled water, assuming that $1 \mathrm{ml}$ of water is equivalent to 1 gram. The collected blood was pooled and washed with KAv-1 medium (Kuwana et al., 1996). Avian PGCs were readily distinguishable from blood cells by their remarkably large size and the presence of considerable numbers of refractive granules (lipids) in the cytoplasm, as observed using phase contrast microscopy (Fujimoto et al., 1976 ; Kuwana, 1993). The PGCs were picked up and counted every developmental stage by hand with a fine glass micropipette (about $45 \mu \mathrm{m}$ outer diameter) from the collected cell suspension. The statistical analysis of differences in the PGC numbers at every developmental stage between KD and WL was performed by Student's t test. A probability of less than 0.05 was considered significant. All the collected PGCs were suspended in fetal bovine serum (FCS, JRH Biosciences, KS, USA) containing 10\% dimethyl sulfoxide (DMSO, Wako Pure Chemical Industries, Ltd., Osaka, Japan) at $4{ }^{\circ} \mathrm{C}$. The cell suspension was placed in a $1.5 \mathrm{ml}$ cryotube (Nalgene, New York, USA). The cryotube was then placed in a freezing vessel (Bicell ; Nihon Freezer, Tokyo, Japan) and kept at $-80^{\circ} \mathrm{C}$ for $12 \mathrm{hrs}$. During the freezing process, the temperature of the freezing vessel decreased by $1^{\circ} \mathrm{C} \min ^{-1}$. The cryotube was then placed in liquid nitrogen $\left(-196^{\circ} \mathrm{C}\right)$ and stored for 1-6 months until injection.

\section{Transplantaion of PGCs}

WL recipient embryos were incubated for 52-54 hrs under the same conditions as described above to obtain stages 12-14 (Hamburger and Hamilton, 1951). Before injection, a window (about 1.5-2.0 $\mathrm{cm}$ diameter) was opened at the sharp end of the WL eggshell. The stocked cryotube was taken out of liquid nitrogen and immediately put into water at $4{ }^{\circ} \mathrm{C}$. After thawing, the cell suspension was diluted and washed with KAv-1 medium. The cells were centrifuged at $200 \mathrm{~g}$ for $5 \mathrm{~min}$, suspended in a small volume of KAv-1 medium (up to $20 \mu \mathrm{l}$ ) and placed in the center of a plastic dish (Falcon No. 1008, Becton Dickinson Co., Lincoln Park, NJ). A certain number of frozen-thawed KD PGCs were picked up by a fine glass micropipette (about $45 \mu \mathrm{m}$ outer diameter) and injected into the peripheral vein of WL embryos. The volume of the inoculum was $1.5-2.0 \mu l$. Injection was terminated by inserting KAv-1 medium bubbles placed behind the inoculum within the needle into the blood vessel (Yasuda et al., 1992). This prevented haemorrhages from the point of injection. The window in the eggshell was sealed with Scotch brand no. 800 tape (3M, St Paul, MN, USA) after injection, and the manipulated embryos were further incubated at $38^{\circ} \mathrm{C}$ in the incubator up to hatching.

\section{Production of Donor-derived Offspring}

The WL chickens injected with KD PGCs (putative germline chimeras) that survived to sexual maturity were mated with KD by artificial insemination and the feather color of their offspring was examined. Since WL is homozygous dominant for the pigment inhibitor gene (I/I), black offspring indicated that the offspring was derived from donor PGCs of KD, whereas white offspring with small patches of black pigmentation indicated that the offspring was derived from PGCs of WL. Black offspring were raised and examined to determine whether or not they had normal reproductive performance.

\section{Results}

We collected and counted PGCs circulating in the bloodstream at every developmental stage from KD and WL embryos, respectively (Fig. 2). No apparent morphological differences were observed between the PGCs obtained from KD and WL embryos. The numbers of PGCs per $1 \mu \mathrm{l}$ blood in the KD embryos were $7.9 \pm 3.1,12.1 \pm 3.8,7.3 \pm 1.2$ and $3.6 \pm 1.0$ at stages $13,14,15$ and 16 (Hamberger and Hamilton, 1951), respectively. On the other hand, those in the WL embryos were $13.5 \pm 3.6$, $14.0 \pm 1.4,14.5 \pm 1.4$ and $8.0 \pm 1.4$, respectively. There were significant differences in the number of PGCs per embryo recovered from the bloodstream between KD and WL embryos at stages 15 and 16 ( $P<0.05$, Student's t test).

The conditions of survival and hatching of the WL recipient chicken embryos following the injection of 60-232 KD PGCs into the embryonic blood- 


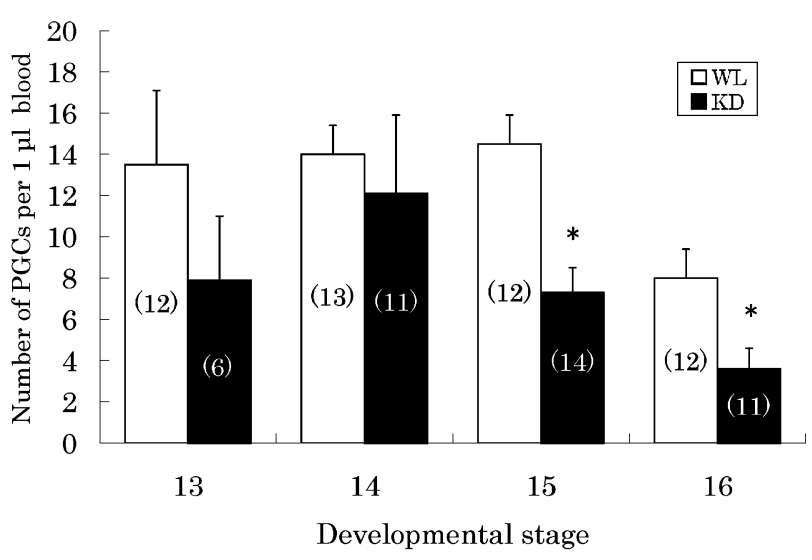

Fig. 2. The number of KD and WL PGCs per $1 \mu l$ (Mean \pm S.E.) embryonic blood at the every developmental stage. Asterisks indicate significant differences between KD and WL $(\mathrm{P}<0.05)$. Numbers in parenthesis are the numbers of embryos. Developmental stages were determined according to the criteria of Hamberger and Hamilton (1951).

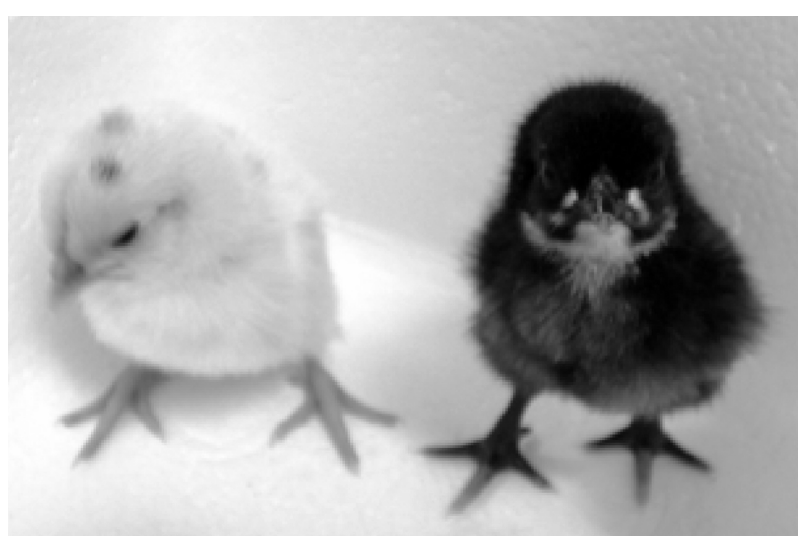

Fig. 3. Phenotypes of offspring from the female germline chimeras. Black offspring (right) indicated that the offspring was derived from donor PGCs of $\mathrm{KD}$, whereas white offspring with small patches of black pigmentation (the left) indicated that the offspring was derived PGCs of WL.

Table 1. The conditions of survival and hatching of WL recipient chicken embryos injected with KD PGCs

\begin{tabular}{ccccc}
\hline $\begin{array}{c}\text { ID of } \\
\text { embryo }\end{array}$ & $\begin{array}{c}\text { The number of } \\
\text { injected PGCs }\end{array}$ & $\begin{array}{c}\text { Developmental } \\
\text { stage at injection }\end{array}$ & Viability & $\begin{array}{c}\text { Sexing } \\
\text { after maturity }\end{array}$ \\
\hline E01 & 60 & 13 & Died on Day & \\
E02 & 100 & 12 & Hatched & Female \\
E03 & 100 & 12 & Hatched & Female \\
E04 & 100 & 12 & Hatched & Female \\
E05 & 100 & 13 & Died on Day 20 & \\
E06 & 100 & 13 & Died on Day 21 & Female \\
E07 & 200 & 13 & Hatched & Male \\
E08 & 200 & 13 & Died on Day 20 & Female \\
E09 & 200 & $13-14$ & Hatched & Female \\
E10 & 200 & $13-14$ & Died on Day 10 & \\
E11 & 200 & 14 & Hatched & Hatched \\
E12 & 232 & 13 & Died on Day 16 & \\
E13 & 232 & 13 & & \\
\hline
\end{tabular}

Developmental stages were determined according to the criteria of Hamberger and Hamilton (1951).

stream are shown in Table 1. The viability of embryos tended to decrease just before hatching, but the hatching rates were $53.8 \%(7 / 13)$. We were able to obtain one male (E09) and six females (E02, E03, E04, E07, E11 and E12) as putative germline chimeras in this study.

The six WL female chickens injected with PGCs of $\mathrm{KD}$ that survived to sexual maturity were mated with KD by artificial insemination. As the feather color of their offspring was examined, we obtained some black offspring which indicated that these offspring were derived from donor PGCs of KD (Fig. 3). As shown in Table 2, the frequency of production of stable KD-WL germline chimeras 
Table 2. Progeny test of female putative germline chimeras transferred from KD PGCs

\begin{tabular}{ccccccccc}
\hline \hline $\begin{array}{c}\text { ID of } \\
\text { embryo }\end{array}$ & $\begin{array}{c}\text { The number } \\
\text { of injected } \\
\text { PGCs }\end{array}$ & $\begin{array}{c}\text { No. of eggs } \\
\text { obtained }\end{array}$ & $\begin{array}{c}\text { No. of } \\
\text { non-fertilized } \\
\text { eggs }\end{array}$ & $\begin{array}{c}\text { The rate of } \\
\text { fertilization } \\
(\%)\end{array}$ & $\begin{array}{c}\text { No. of } \\
\text { chicks } \\
\text { hatched }\end{array}$ & $\begin{array}{c}\text { No. of } \\
\text { white } \\
\text { chicks }\end{array}$ & $\begin{array}{c}\text { No. of } \\
\text { black } \\
\text { chicks }\end{array}$ & $\begin{array}{c}\text { Frequency of } \\
\text { black chicks } \\
(\%)\end{array}$ \\
\hline E02 & 100 & 192 & 13 & 93.2 & 146 & 145 & 1 & 0.7 \\
E03 & 100 & 188 & 22 & 88.3 & 145 & 145 & 0 & 0 \\
E04 & 100 & 189 & 78 & 58.7 & 76 & 72 & 4 & 5.3 \\
E07 & 200 & 245 & 4 & 98.4 & 222 & 222 & 0 & 0 \\
E11 & 200 & 249 & 4 & 98.4 & 218 & 217 & 1 & 0.5 \\
E12 & 232 & 196 & 6 & 96.9 & 137 & 135 & 2 & 1.5 \\
\hline
\end{tabular}

Test periods lasted for 10 months.

which produced black chicks was $66.7 \%(4 / 6$ : E 02, E04, E11 and E12). However, the average rate of the KD-derived offspring obtained from the four KD-WL female germline chimeras was only $1.4 \%$ (8 out of all 577 hatched chicks) and the maximum was $5.3 \%$ (E04). We were not able to perform the progeny test for one male putative germline chimera (E09; Table 1) by mating with a KD female by artificial insemination, because KD females are very rare and produce few eggs (approximately 50 eggs per a year).

Eight donor-derived offspring (KD) obtained from the four KD-WL female germline chimeras (E02, E04, E11 and E12) reached maturity, and both fertility and hatchability were normal.

\section{Discussion}

We were able to obtain germline chimeras between $\mathrm{KD}$, as a model of an endangered chicken breed, and WL as a common poultry. In the present study, we realized the speculation of Tajima et al. (1993) that the transplanting of PGCs could be applied to augment populations of endangered chicken breeds under natural mating conditions. Although it has already become possible to conserve genetic resources in chickens by preserving PGCs in liquid nitrogen (Naito et al., 1994 b), there is still the remaining, important question of how to obtain offspring with a high efficiency from germline chimeras.

The number of PGCs in the bloodstream of stage $15 \mathrm{WL}$ embryos was significantly over 1.9 times higher than that in KD (Fig. 2). One WL chicken produces approximately 250-plus eggs per a year, while one KD produces few eggs. Regardless of the number of donor PGCs injected into the recipient embryos, however, we were able to obtain germline chimeras in this study (Table 2). Naito et al. (1999) reported that the efficiency of obtaining donor-derived offspring from the germline chimeric chickens was enhanced by increasing the number of PGCs injected into recipient embryos, although these correlation coefficients were not significant. Some PGCs in the early chick embryo do not migrate into the gonadal primordium but locate in extragonadal sites (Nakamura et al., 1988). Since all donor-derived PGCs injected into the bloodstream can not settle at the gonadal anlage, further studies are needed to elucidate how many PGCs are sufficient to establish germline chimeras.

There is a possibility that the apparent dominance of WL over KD involves competition for germ cell proliferation in the recipient gonads. It is important to understand how to reduce endogenous PGCs of recipient embryos in order to enhance the efficiency of establishing germline chimera. Several methods have been attempted to eliminate or reduce endogenous PGCs of recipient embryos : ultraviolet irradiation (Aige-Gil and Simkiss, 1991), laser irradiation (Mims and McKinnell, 1971), application of buslfan (Hallett and Wentworth, 1991; Vick et al., 1993), application of concanavalin A (Al-Thani and Simkiss, 1991) and excision of the germinal crescent region (McCarry and Abbott, 1982). Naito et al. (1994 a) reported that removing blood from the recipient embryos prior to PGC injection seems to be the most effective of the present methods for the production of germline chimeras, since it is simple to perform and causes less damage to embryonic development. Because 
we did not remove blood from the recipient embryos in this study following Naito's procedure, the transmission rate of donor-derived gametes was very low in germline chimeras. This suggested that drawing blood from recipient embryos prior to PGC injection might be more effective for WL than $\mathrm{KD}$. There is controversy as to which procedure enhances the frequency of donor-derived offspring.

PGCs have already had their sex determined, since they are the progenitor cells of gametes as oocytes in females or sperm in males, respectively. Naito et al. (1999) elucidated that the percentages of chimeric chickens that produced donor-derived offspring were significantly higher for same-sex combinations of donor and recipient embryos compared with different-sex combinations. In chickens, a rapid protocol for sexing embryos has been developed : since the male is the homogametic sex (ZZ), while the female is the heterogametic sex (ZW) in avian species, sequences of primers for detecting the W chromosome-specific repeating sequences has been developed (Clinton, 1994). The sex identification and fitness of donor and recipient embryos might enhance the frequency of donorderived offspring.

Further studies are required on the frequency of germline transmission of donor PGCs, but the other remaining areas to be studied are : methods for the in vitro proliferation of PGCs and the production of interspecific germline chimeras using PGCs. Recently, we revealed that embryonic fibroblast-conditioned medium enhances the viability and proliferation of chicken PGCs in suspension culture (Kawashima et al., 2002). From the results of the previous report, we will attempt to establish a long-term culture of avian PGCs. On the other hand, once adult interspecific chimeras become available, there will be a chance to recover endangered or even extinct species. In fish, PGCs can be transplanted between two different salmonid species, with the subsequent production of xenogenic, donor-derived offspring (Takeuchi et al., 2004). However, the above procedures may be a long way from becoming a reality in Aves. But, provided similar techniques can be performed for endangered avian species, we may further proceed.

In conclusion, we showed that the technique for producing germline chimeras with PGCs between the same kinds of breed might be applied for pre- serving foundation stock that may be lost through unexpected disease or other events.

\section{Acknowledgments}

We would like to thank the staff of the Laboratory of Intellectual Fundamentals for Environmental Studies, National Institute for Environmental Studies for taking care of the birds. This study was supported by the Special Coordination Fund from the Ministry of Education, Culture, Sports, Science and Technology, Japan.

\section{References}

Aige-Gil V and Simkiss K. Sterilising embryos for transgenic chimaeras. British Poultry Science, 32 : 427-438. 1991.

Al-Thani R and Simkiss K. Effects of an acute in vivo application of concanavalin $\mathrm{A}$ on the migration of avian primordial germ cells. Protoplasma, $161: 52-57$. 1991.

Clinton M. A rapid protocol for sexing chick embryos (Gallus g. domesticus). Animal Genetics, 25 : 361362. 1994.

Fujimoto T, Ukeshima A and Kiyofuji R. The origin, migration and morphology of the primordial germ cells in the chick embryo. The Anatomical Record, 185 : 139-145. 1976.

Hallett JS and Wentworth BC. The effects of busulfan on gonadal differentiation and development in Japanese quail (Coturnix coturnix japonica). Poultry Science, 70 : 1619-1623. 1991.

Hamburger V and Hamilton HL. A series of normal stages in the development of the chick embryo. Journal of Morphology, 88 : 49-92. 1951.

Kawashima T, Kano K, Kannan Y, Tamaki Y and Hashimoto H. Embryonic fibroblast-conditioned medium enhances viability and proliferation of chick circulating primordial germ cells (cPGCs) in suspension culture. The Journal of Reproduction and Development, 48 : 143-150. 2002.

Kuwana T. Migration of avian primordial germ cells toward the gonadal anlage. Development, Growth and Differentiation, 35 : 237-243. 1993.

Kuwana T, Hashimoto K, Nakanishi A, Yasuda Y, Tajima A and Naito M. Long-term culture of avian embryonic cells in vitro. The International Journal of Developmental Biology, 40 : 1061-1064. 1996.

Kuwana T and Rogulska T. Migratory mechanisms of chick primordial germ cells toward gonadal anlage. Cellular and Molecular Biology, 45 : 725-736. 1999.

Matsuzaki M. Keeping and breeding of native fowl for chicken meat in Kumamoto. Japanese Poultry Science (in Japanese), $38: 182-189.2001$.

McCarrey JR and Abbott UK. Functional differentiation of chick gonads following depletion of primordial germ cells. Journal of Embryology and Experimental Morphology, 68 : 161-174. 1982. 
Mims MF and McKinnell RG. Laser irradiation of the chick embryo germinal crescent. Journal of Embryology and Experimental Morphology, 26 : 31-36. 1971.

Naito M, Tajima A, Yasuda Y and Kuwana T. Production of germline chimeric chickens, with high transmission rate of donor-derived gametes, produced by transfer of primordial germ cells. Molecular Reproduction and Development, 39 : 153-161. 1994 a.

Naito M, Tajima A, Tagami T, Yasuda Y and Kuwana T. Preservation of chick primordial germ cells in liquid nitrogen and subsequent production of viable offspring. Journal of Reproduction and Fertility, 102 : 321-325. 1994 b.

Naito M, Matsubara Y, Harumi T, Tagami T, Kagami H, Sakurai $\mathbf{M}$ and Kuwana T. Differentiation of donor primordial germ cells into functional gametes in the gonads of mixed-sex germline chimaeric chickens produced by transfer of primordial germ cells isolated from embryonic blood. Journal of Reproduction and Fertility, 117 : 291-298. 1999.

Nakamura M, Kuwana T, Miyayama Y and Fujimoto T.
Extragonadal distribution of primordial germ cells in the early chick embryo. The Anatomical Record, 222 : 90-94. 1988.

Swift $\mathrm{CH}$. Origin and early history of the primordial germ cells in the chick. The American Journal of Anatomy, 15 : 483-516. 1914.

Takeuchi Y, Yoshizaki G and Takeuchi T. Surrogate broodstock produces salmonids. Nature, 430 : 629630. 2004.

Tajima A, Naito M, Yasuda Y and Kuwana T. Production of germ line chimera by transfer of primordial germ cells in the domestic chicken (Gallus domesticus). Theriogenology, 40 : 509-519. 1993.

Vick L, Luke G and Simkiss K. Germ-line chimaeras can produce both strains of fowl with high efficiency after partial sterilization. Journal of Reproduction and Fertility, $98: 637-641.1993$.

Yasuda Y, Tajima A, Fujimoto T and Kuwana T. A method to obtain avian germ-line chimaeras using isolated primordial germ cells. Journal of Reproduction and Fertility, 96 : 521-528. 1992. 